\title{
tic\&société
}

Vol. 5, n²-3 | 2e sem. 2011 / 1er sem. 2012

Les TICs dans les pays des Suds

\section{De la domination politique à la domination} économique : une histoire des télécommunications au Sénégal

\section{Olivier Sagna}

\section{OpenEdition \\ Journals}

Édition électronique

URL : http://journals.openedition.org/ticetsociete/1030

DOI : 10.4000/ticetsociete. 1030

Éditeur

Association ARTIC

\section{Référence électronique}

Olivier Sagna, « De la domination politique à la domination économique : une histoire des

télécommunications au Sénégal », tic\&société [En ligne], Vol. 5, n²-3 | 2e sem. 2011 / 1er sem. 2012,

mis en ligne le 18 juin 2012, consulté le 19 avril 2019. URL : http://journals.openedition.org/

ticetsociete/1030; DOI : 10.4000/ticetsociete.1030 


\title{
De la domination politique à la domination économique : une histoire des télécommunications au Sénégal
}

\author{
Olivier SAGNA \\ Maître de conférences \\ École de bibliothécaires, archivistes et documentalistes (EBAD), \\ Université Cheikh Anta Diop de Dakar (UCAD) \\ EBAD, BP 3252, Dakar, Sénégal \\ olivier.sagna@ebad.ucad.sn
}

Olivier Sagna est Maître de conférences à l'Ecole de bibliothécaires, archivistes et documentalistes (EBAD) de I'Université Cheikh Anta Diop de Dakar (Sénégal) où il enseigne les sciences de l'information depuis 1988. Historien de formation, il est titulaire d'un doctorat en histoire et du diplôme de I'Institut national des techniques de la documentation (INTD) du Conservatoire national des arts et métiers (CNAM) de Paris. Ses recherches et publications portent sur le développement de la société de l'information en Afrique et s'intéressent notamment aux politiques publiques, à l'économie des télécommunications et des TIC, aux usages et à l'utilisation des TIC dans l'enseignement supérieur et la recherche. En tant qu'expert, il a également été impliqué dans l'élaboration de stratégies relatives à la société de l'information et au développement de l'économie numérique. 


\title{
De la domination politique à la domination économique : une histoire des télécommunications au Sénégal
}

\begin{abstract}
Résumé : Le télégraphe, premier des moyens de télécommunication modernes, fut introduit au Sénégal dans la seconde moitié du $\mathrm{XIX}^{\mathrm{e}}$ siècle pour répondre aux besoins de la France en matière de contrôle administratif et militaire, d'exploitation économique et de communication dans le cadre de l'entreprise coloniale. L'architecture du réseau, polarisée autour de quelques villes au détriment des zones rurales, servira de matrice au réseau téléphonique. Après l'indépendance, les télécommunications se verront accorder un rôle critique dans la politique de développement économique et social et se développeront au point de devenir un des principaux piliers de l'économie. Cependant, les politiques de libéralisation et de privatisation feront passer ce secteur particulièrement stratégique et rentable sous la coupe des multinationales étrangères, refermant ainsi la courte parenthèse durant laquelle il fut géré souverainement, faisant perdre par la même occasion au Sénégal la maîtrise d'un important levier de développement.
\end{abstract}

Mots clés: Télécommunications, Politique, Economie, TIC, Histoire, Sénégal.

\begin{abstract}
The telegraph, the first modern telecommunications tool, was introduced in Senegal during the second half of the nineteenth century to ease administrative and military control, economic exploitation and communication with France, as part of the colonial enterprise. The network architecture, concentrated around few cities to the detriment of rural areas, later formed the basis of the telephone infrastructure. After independence, the telecommunications sector was assigned a critical role in economic and social development and it soon developed to become a mainstay of the Senegalese economy. However, liberalization and privatization policies have put this particularly strategic and profitable sector under the control of foreign multinational firms shortly after, thus causing Senegal the loss of mastery of an important development tool.
\end{abstract}

Keywords: Telecommunications, Politics, Economy, History, Senegal. 
De la domination politique à la domination économique : une histoire des télécommunications au Sénégal

Resumen : El telégrafo, la primera de las telecomunicaciones modernas, se introdujo en Senegal en la segunda mitad del siglo XIX, para cumplir con Francia en materia de control administrativo y militar, la explotación económica y la comunicación como parte de la empresa colonial . La arquitectura de la red, polarizada en torno a unas pocas ciudades en detrimento de las zonas rurales, que sirvira como matriz a la red telefónica. Después de la independencia, a las telecomunicaciones se les dará un papel fundamental en la política de desarrollo económico y social y crecerán para convertirse en uno de los fundamentos de la economía. Sin embargo, las políticas de liberalización y privatización aumentará el sector particularmente estratégico y rentable bajo el control de las multinacionales extranjeras, cerrando así el breve interludio durante el cual que dirigido con soberamía, haciendo perder al mismo tiempo, a Senegal un importante punto de apoyo para el desarrollo.

Palabras clave: Comunicación, Política, Economía, TIC, Historia, Sénégal. 
Olivier SAGNA

Le passé est toujours présent

Maurice Maeterlinck

\section{Introduction}

Dans l'entendement du grand public comme dans l'esprit de nombre d'analystes qui s'intéressent aux problématiques liées à l'émergence de la société de l'information, les technologies de l'information et de la communication (TIC) évoquent, par essence, le progrès scientifique et technique, l'innovation, la nouveauté quand ce ne sont pas les promesses du futur. Ce faisant, les uns et les autres ont parfois tendance à oublier que les TIC possèdent également une histoire qu'il est important de connaître car elle détermine souvent le présent. Ainsi, la configuration du réseau de télécommunications, le rôle que jouent les TIC dans l'économie ainsi que la place que l'État leur accorde dans la politique de développement d'un pays ne peuvent se comprendre sans se référer à l'histoire de leur déploiement. Carrefour des routes maritimes reliant l'Europe, l'Afrique et l'Amérique latine, escale aérienne entre l'Afrique et les Amériques, finistère de l'Afrique de l'Ouest, capitale de l'Afrique occidentale française (AOF), siège de nombreuses organisations internationales, pays touristique, le Sénégal est depuis longtemps impliqué dans une multitude de relations dans lesquelles les télécommunications ont occupé, et continuent d'occuper, une place prépondérante. Nous appuyant sur une documentation diversifiée mêlant travaux académiques, articles et ouvrages portant sur la question, nous proposons de retracer la perspective historique dans laquelle s'inscrit le développement des télécommunications au Sénégal de la fin du $\mathrm{XIX}^{\mathrm{e}}$ siècle à ce début de $\mathrm{XXI}^{\mathrm{e}}$ siècle en mettant l'accent sur les permanences et les ruptures.

\section{1. À l'origine fut le télégraphe}

Jusqu'à l'occupation des territoires correspondant au Sénégal contemporain par les Européens, il n'existe pas d'administration postale chargée de l'acheminement du courrier. À l'échelle des différentes concentrations humaines, l'information est transmise par le biais de messagers à pied, voire, pour l'annonce de certains évènements, par le biais du tambour. Sur les grandes distances, les nouvelles ordinaires circulent par l'intermédiaire des voyageurs et des commerçants tandis que les pouvoirs en place recourent à des cavaliers afin de véhiculer les informations liées au contrôle des territoires sur lesquels ils exercent leur souveraineté [Niang, 1977, p. 3]. 
De la domination politique à la domination économique : une histoire des télécommunications au Sénégal

Le premier système de communication moderne est mis en place au XVII siècle durant la période précédant la conquête coloniale. En effet, les premières relations postales avec la France sont établies en 1626, date à laquelle les navires des commerçants dieppois et rouennais touchent les côtes sénégalaises. Ce n'est qu'après l'installation d'un représentant officiel du Roi de France, vers 1782, puis le début de l'occupation de l'intérieur du pays, qu'un service postal embryonnaire est créé. Cependant, il faut attendre 1879 pour qu'une véritable organisation postale soit mise en place au Sénégal [Gouvernement général de l'AOF, 1907, p. 111]. Son principal objectif est de relier Saint-Louis aux différents points du territoire jouant un rôle administratif, militaire ou économique. Elle repose principalement sur le courrier piéton qui fonctionne grâce à des porteurs convoyant des sacs de dépêches sur des distances de trente à trente-cinq kilomètres [Niang, 1977, p. 15]. Sur certains axes, le transport du courrier est assuré par d'autres moyens tels que le chemin de fer entre Saint-Louis et Dakar, les bateaux à vapeur sur les voies fluviales entre Dakar et Gorée, la poste par chameaux entre Saint-Louis et Gandiole ${ }^{1}$ ainsi que le train des équipages dont la vocation est essentiellement militaire. Les communications avec la France, qui jouent un rôle critique puisque c'est dans la métropole que se prennent toutes les décisions d'importance relatives à la colonie, sont assurées par des navires opérant principalement à partir des ports de Bordeaux et de Marseille. Le dénominateur commun à tous ces systèmes de communication est leur lenteur et leur manque de fiabilité qui font que le courrier met des jours, des semaines, voire des mois, avant d'arriver à destination lorsqu'il ne se détériore pas ou ne se perd pas en cours de route.

Afin de remédier à ce problème, dans la seconde moitié du $\mathrm{XIX}^{\mathrm{e}}$ siècle, l'Administration coloniale décide de recourir au télégraphe électrique dont la première ligne a été inaugurée en France en 1844 [Figuier, 1849, p. 618]. Le télégraphe, qui à l'origine était un moyen de communication militaire, est en effet devenu un puissant instrument de gouvernement au service de l'administration [Field, 1994, p. 344]. II n'est donc pas surprenant que les autorités françaises, confrontées à des problèmes de communication et d'administration du territoire sénégalais, décident de l'utiliser dans le cadre de l'entreprise coloniale.

C'est ainsi qu'en 1859, une ligne télégraphique expérimentale est construite entre Saint-Louis et Gandiole. Le service connaît un tel succès auprès des autorités politiques comme des milieux économiques que l'Administration coloniale décide de généraliser son utilisation. Le premier chantier d'envergure porte sur la construction, entre 1861 et 1862, d'une ligne télégraphique reliant

\footnotetext{
${ }^{1}$ Situé sur la côte, Gandiole est un point où sont entreposées les marchandises destinées à être
} acheminées dans l'intérieur du pays. 
Olivier SAGNA

Saint-Louis à Gorée en passant par Dakar. Ces trois villes sont, en effet, avec Rufisque, au cœur du dispositif colonial français et constitueront, à partir de 1872 pour Saint-Louis et Gorée puis à partir de 1880 pour Rufisque et 1887 pour Dakar, les célèbres "Quatre communes " dont les habitants ont la particularité de posséder la citoyenneté française [Wesley Johnson, 1991]. Progressivement, les régions du Fleuve, de la Petite côte, du Sine-Saloum, du Baol et de la Casamance sont équipées, au point qu'en 1900 le réseau télégraphique est long de 3196 kilomètres et couvre tous les points du territoire sénégalais ayant une importance administrative, militaire ou économique [Niang, 1977, p.119]

Les bureaux de poste disposant du télégraphe sont habilités à envoyer et recevoir des télégrammes, tant officiels que privés, mais l'Administration coloniale en est le principal utilisateur. Elle bénéficie d'un droit de franchise qui lui permet d'envoyer gratuitement des dépêches officielles et lui donne la priorité pour l'envoi des télégrammes. Ce régime préférentiel fait que les fonctionnaires privilégient l'envoi de télégrammes par rapport au courrier postal avec pour conséquence de peser négativement sur la rentabilité économique du télégraphe. Cependant, à partir de 1919, l'Administration coloniale décide de privilégier l'exploitation commerciale du télégraphe ce qui la conduit à supprimer le droit de franchise dont elle bénéficiait auparavant et l'oblige désormais à payer pour l'utilisation des services télégraphiques [Thiaw, 1995, p.9].

Le déploiement du télégraphe s'inscrit dans la logique de l'entreprise coloniale qui vise, d'une part, à s'assurer le contrôle militaro-administratif du territoire en vue d'y faire régner l'ordre colonial et, d'autre part, à faciliter les communications permettant d'exploiter ses ressources. De ce fait, en dehors des fonctionnaires et des militaires coloniaux, les principaux utilisateurs du télégraphe sont les commerçants et les métis qui constituent un groupe social très influent à Saint-Louis [Mbaye, 1980] et à Gorée. Quant aux autochtones, ils en sont des utilisateurs marginaux, maintenus qu'ils sont à la périphérie de la société coloniale par le Code de l'indigénat. Bien qu'ouverte aux usages privés, l'utilisation du télégraphe reste contraignante du fait de la nécessité d'encoder et de décoder les messages, ce qui limite l'expansion de cet outil.

\section{La connexion au système mondial capitaliste}

La modernisation des communications avec l'extérieur prend place dans le dernier quart du $\mathrm{XIX}^{\mathrm{e}}$ siècle avec la construction de câbles sous-marins reliant le Sénégal au reste du monde. Le premier est installé en 1885 par la Spanish Submarine Telegraph Company qui, dans le cadre de la convention francoespagnole du 2 mai 1884, pose un câble reliant Cadix (Espagne) à Yoff 
De la domination politique à la domination économique : une histoire des télécommunications au Sénégal

(Sénégal) en passant par Ténériffe (Canaries) et Saint Louis. En 1886, ce câble est prolongé jusqu'à Luanda (Angola) par la West African Telegraph Company [Sy, 1996, p. 58], permettant à la Guinée, la Côte d'Ivoire, le Dahomey et le Gabon d'être connectés à ce réseau ${ }^{2}$. En 1892, un troisième câble allant de Dakar à Recife (Brésil) est déployé par la South American Cable Company, reliant ainsi le Sénégal à l'Amérique latine [AACSM, 2006]. Enfin, un câble allant de Brest (France) à Dakar est construit en 1905 par la Société industrielle des téléphones (SIT) [Lombard, 2006, p. 69], assurant une certaine redondance au système et permettant surtout à la France de s'affranchir du monopole britannique sur les câbles sous-marins [Zimmermann, 1900, p. 469].

La construction d'un réseau télégraphique local et de liens permanents entre la France et le Sénégal symbolise la pérennisation de la connexion de l'Afrique au système capitaliste mondial. Ces câbles arriment en effet, au propre comme au figuré, le Sénégal, pays de la périphérie, au cœur du système capitaliste, à l'époque localisé en Europe de l'Ouest. Ils contribuent notamment à organiser, dominer, exploiter et influencer son économie [Wallerstein, 1985] au profit de la métropole coloniale. En effet, bien qu'initié au milieu du $X V^{e}$ siècle avec l'arrivée des premiers navigateurs portugais, ce processus de domination n'a véritablement pris forme qu'à partir du $\mathrm{XVII}{ }^{\mathrm{e}}$ siècle avec le développement $\mathrm{du}$ commerce triangulaire [Ly, 1958]. II était cependant fragile car les communications dépendaient uniquement des navires assurant la liaison avec la métropole. Cet amarrage du Sénégal à la France se met en place durant la période qui précède la naissance de l'Afrique occidentale française (AOF) ${ }^{3}$, entité administrative dont l'avènement marque la fin de la " conquête coloniale " suite à l'anéantissement des dernières résistances armées et le triomphe de l'ordre colonial qui vise à "La mise en valeur des colonies " ${ }^{4}$. L'importance prise par les télécommunications est d'ailleurs telle que les autorités coloniales créent en 1903 une Inspection des postes et télégraphes de l'AOF. Comme pour bien marquer les rapports asymétriques existant entre le centre et la périphérie du système colonial, la gestion des câbles sous-marins reliant le Sénégal au reste du monde relève de la métropole [Desbois, 2000, p. 135] tandis que le réseau intérieur desservant le Sénégal est du ressort de l'administration locale.

\footnotetext{
${ }^{2}$ Compte tenu de l'importance stratégique de ces liaisons, l'administration des postes et télégraphes entreprendra des pourparlers avec la West African Telegraph Company qui aboutiront, en 1902, au rachat des câbles sous-marins aboutissant dans les territoires sous contrôle de la France.

${ }^{3}$ Créée en 1895 avec Dakar comme capitale, I'AOF regroupe la Guinée, la Côte d'Ivoire, le Soudan et le Sénégal auxquels viendront s'ajouter en 1904 le Dahomey, la Mauritanie et le Niger puis la Haute Volta et le Togo en 1919.

${ }^{4}$ Titre de l'ouvrage publié en 1923 par Albert Sarraut, ministre des Colonies de 1920 à 1924.
} 


\section{Du téléphone aux télécommunications spatiales}

Exploité commercialement en France à partir de 1885 par la Société générale des téléphones (SGT) et transformé en monopole d'État à partir de 1889, le téléphone est introduit au Sénégal en 1901. À l'époque, le parc d'utilisateurs se limite à une centaine d'abonnés répartis entre Saint-Louis, Dakar et Rufisque [Lemesle, 2002, p. 39]. Les investissements publics étant entièrement à la charge des colonies, le réseau téléphonique reste à un stade embryonnaire pendant plusieurs décennies. Une première rupture s'opère en 1943 avec la construction du central téléphonique automatique de Dakar-Ponty d'une capacité de 900 lignes. Cependant, ce n'est qu'après la Seconde guerre mondiale, grâce notamment au Fonds d'investissement pour le développement économique et social des territoires d'outre-mer (FIDES), que des moyens significatifs sont mobilisés en vue de développer le réseau téléphonique. C'est ainsi que le central de Dakar-Ponty voit ses capacités passer à 2000 lignes en 1948, puis à 3000 en 1950 auxquelles viennent s'ajouter 3000 lignes supplémentaires en 1953 avec la construction du central téléphonique de Dakar-Médina. La dernière innovation de l'ère coloniale consiste en l'introduction du télex en 1957 [Fall, 1964, p. 489]. Si des progrès notables ont été faits pour développer les télécommunications au Sénégal, force est de constater que le déploiement de l'infrastructure s'est, pour l'essentiel, limité aux concentrations urbaines et principalement à Dakar.

En 1960, lorsque le Sénégal accède à la souveraineté internationale, les services de télécommunications passent sous la tutelle de l'Office des postes et télécommunications $(\mathrm{OPT})^{5}$ et le pays se dote d'un Comité national de coordination des télécommunications (CNCT). Les statistiques établies l'année suivante indiquent que le réseau téléphonique totalise quelques 9857 abonnés et comporte 109 lignes à usage public sous forme de cabines téléphoniques installées dans les bureaux de poste pour une population de 3557989 habitants. La répartition géographique des abonnés révèle que $70 \%$ des lignes principales sont concentrées à Dakar et que $89 \%$ des lignes téléphoniques du pays sont situées dans les centres urbains [Sy, 1996, p. 76]. Malgré les nouvelles dynamiques politiques, économiques, culturelles et sociales nées de l'indépendance, le nombre d'abonnés progresse lentement et, en 1968, le Sénégal, dont la population s'élève désormais à 4195353 habitants, atteint tout juste le seuil des 10000 abonnés, dont 7500 "privés " et 2500 « administratifs » [Royer, 1969, p.43]. En termes de répartition géographique, la région du Cap-Vert, qui abrite la capitale du pays, concentre $81 \%$ des lignes privées et $47 \%$ des lignes utilisées par l'administration. La même polarisation se

\footnotetext{
${ }^{5}$ Ordonnance $n^{\circ}$ 60-22 du 3 octobre 1960 créant l'Office des postes et télécommunications.
} 
De la domination politique à la domination économique : une histoire des télécommunications au Sénégal

retrouve à l'échelle des capitales régionales dont la plupart détiennent entre les trois quarts et la moitié des lignes installées dans leurs limites administratives [Royer, 1969, p.45]. La configuration du réseau est telle que les zones qui n'abritent pas d'activités économiques d'importance nationale s'en trouvent exclues et sa cartographie montre qu'il couvre principalement la façade atlantique qui constitue en quelque sorte le pays « utile ».

Paradoxalement, les télécommunications internationales sont toujours gérées par l'ancienne métropole via France Câbles et Radio $(F C R)^{6}$. Le schéma, également mis en place dans les autres pays anciennement sous tutelle française, consiste pour FCR à mettre en place les moyens nécessaires à la création, au développement et à l'exploitation des télécommunications internationales, en assumant toutes les charges liées aux investissements comme aux frais d'exploitation pour, en contrepartie, être rémunéré sur la base d'une quote-part sur les recettes générées par l'exploitation du trafic acheminé, le solde revenant à l'État sénégalais [Hachmanian, 2006].

En 1968, l'État décide de séparer la gestion des télécommunications nationales et internationales en confiant ces dernières à la société TéléSénégal, société d'économie mixte, cogérée par l'Office des postes et télécommunications (OPT) pour le compte du Sénégal et par FCR pour le compte de la France. Ce changement institutionnel est dicté par l'apparition des télécommunications par satellite qui, étant gérées à l'échelle internationale par le consortium Intelsat pour les questions opérationnelles sous la supervision de l'Union internationales des télécommunications (UIT) pour l'allocation des fréquences [Erne, 2007], font de chaque pays le pilote de son propre système. Dans ce cadre, une politique de modernisation des liaisons internationales est entreprise avec la construction, en 1972, de la station terrienne de télécommunications spatiales de Gandoul. Première du genre sur le contient africain, elle conduit, à partir de 1978, à l'automatisation des communications internationales et du télex [Thiaw, 1995, p. 9]. Ce dispositif est complété par la mise en service des câbles sous-marins Antinéa entre le Sénégal et le Maroc en 1977, Fraternité entre le Sénégal et la Côte d'ivoire en 1978, Atlantis 1 entre le Sénégal et le Brésil en 1982 et Atlantis 2 entre le Sénégal et le Portugal en 1982 [UIT, 1998a, pp 12-13].

\footnotetext{
${ }^{6}$ FCR a été créée en 1959 pour permettre à la France de continuer à gérer les télécommunications internationales dans les colonies françaises qui s'acheminaient vers l'indépendance.
} 


\section{L'heure des premières réformes institutionnelles}

La période allant de l'indépendance au milieu des années 80 a vu le réseau de télécommunications s'étendre dans les zones urbaines. De son côté, l'architecture institutionnelle du secteur a peu évolué si l'on excepte la nationalisation de TéléSénégal. Cette décision a été prise suite au constat fait par l'État sénégalais que les télécommunications internationales se développaient fortement sans qu'il n'en retire un grand bénéfice. Le Sénégal a alors entamé des négociations avec la France à l'issue desquelles ses parts dans le capital de TéléSénégal sont passées, dans un premier temps, de $26 \%$ à $51 \%$ en 1976. Finalement, ce processus a abouti à la nationalisation de TéléSénégal en 1981 avec le rachat, sur plusieurs années, et grâce aux bénéfices dégagés par l'opérateur, des $49 \%$ encore détenus par FCR [Kane, 2010, pp. 107-108].

C'est dans ce contexte qu'Alassane Dialy Ndiaye, alors directeur général de TéléSénégal, rédige un rapport à l'attention du Président de la République dans lequel il recommande de réunir les télécommunications nationales et internationales au sein d'une entité unique. Cette réflexion aboutit en 1983 à l'organisation des Journées nationales des télécommunications qui dressent un bilan peu reluisant de la situation (seulement 20500 lignes téléphoniques pour huit millions d'habitants) et concluent à la nécessité de séparer les activités postales de celles de télécommunications. En 1985, l'État lance la première grande réforme de ce secteur qui débouche sur l'éclatement de I'OPT avec la création, d'une part, de l'Office de la poste et de la caisse d'épargne (OPCE) ${ }^{7}$ et, d'autre part, de la Société nationale des télécommunications du Sénégal $(\text { Sonatel) })^{8}$. Par ailleurs, l'État décide de donner la priorité au développement des télécommunications dans le $\mathrm{VII}^{\mathrm{e}}$ Plan de développement économique et social (1985-1989) en fixant quatre objectifs à la Sonatel à savoir (1) développer une infrastructure hautement productive et capable de stimuler l'activité économique nationale, (2) améliorer l'accès aux télécommunications, (3) faciliter le développement des banques de données nationales et (4) susciter l'implantation d'une industrie locale ou régionale des télécommunications [Daffé et Dansokho, 2002, p. 54]. Autre décision prise par les autorités, celle de mettre en œuvre un plan d'urgence et de rattrapage du réseau national des télécommunications afin de le moderniser et d'en étendre la couverture. Cette politique s'inscrit dans le Plan d'action de Lagos pour le développement économique de l'Afrique (1980-

\footnotetext{
${ }^{7}$ Loi $n^{\circ}$ 85-35 du 23 juillet 1985 portant création de l'Office de la poste et de la caisse d'épargne (OPCE).

${ }^{8}$ Loi $\mathrm{n}^{\circ} 85-36$ du 23 juillet 1985 portant création de la Société nationale des télécommunications (Sonatel).
} 
De la domination politique à la domination économique : une histoire des télécommunications au Sénégal

2000), adopté par l'Organisation de l'unité africaine (OUA), qui accorde un rôle primordial aux télécommunications.

\section{La modernisation et la diversification des services}

Cette politique porte ses fruits puisque le nombre d'abonnés à la téléphonie fixe est porté de 23000 en 1985 à 116000 en 1997 pour finalement atteindre les 200000 abonnés en 2000, soit une densité téléphonique de 12 pour 1000 habitants [Daffé et Dansokho, 2002, p. 61], la plus élevée d'Afrique de l'Ouest à l'exception du Cap-Vert. Cependant, la couverture géographique du réseau est toujours aussi inégale avec une concentration de près de $70 \%$ des lignes dans la capitale. En 1991, la Sonatel créé avec France Câbles et Radio (FCR)-une filiale dénommée Télécomplus ${ }^{9}$ dont la vocation est de développer les produits et services liés aux TIC. À partir de 1992, elle expérimente notamment, des "télécentres" offrant des services de téléphonie, de télécopie et de photocopie. L'expérience est un échec car ces structures s'avèrent peu rentables mais elle donne naissance à ce qui deviendra une véritable "success story ", à savoir les télécentres privés [Sagna, 2009]. Lancés en 1993, ils consistent en des espaces gérés et aménagés par des sociétés privées qui, dans le cadre d'un contrat signé avec la Sonatel, sont autorisées à revendre des services de télécommunications (téléphonie et télécopie). Répondant au fort besoin de communication des Sénégalais, leur nombre passe d'un peu plus d'une centaine en 1993 à plus de 25000 en 2006, créant des milliers d'emplois, générant un important chiffre d'affaires et, surtout, contribuant fortement à démocratiser l'accès au téléphone. Cependant, à partir de 2007, concurrencés par la téléphonie mobile, ils perdent en intérêt pour les consommateurs et en rentabilité pour leurs exploitants, ce qui entraîne un mouvement de fermetures massives à tel point qu'en décembre 2011, il en reste un peu moins de trois mille dans l'ensemble du pays [ARTP, 2011a].

D'autres innovations interviennent comme la numérisation complète du réseau de transmission et la mise en service en 1988 de SENPAC, réseau de transmission de données par paquets de type X25 [Sagna, 2008, p. 18]. Cette infrastructure permet de lancer en 1994 les services vidéotex nationaux (Vidéotel) et internationaux (Minitelnet). Cependant, le Minitel ne rencontre guère de succès du fait de la cherté du terminal et des frais de communications

\footnotetext{
${ }^{9}$ Le capital de Télécomplus est détenu à $51 \%$ par la Sonatel et à $49 \%$ par France Câbles et Radio (FCR).
} 
Olivier SAGNA

mais également faute de l'existence d'une masse critique de services utiles ${ }^{10}$. Par contre, le kiosque audiotex (télématique vocale), lancé en 1995 sous le nom d'Infotel, est bien mieux accueilli du fait qu'il ne nécessite pas l'acquisition d'un terminal spécifique, présente une gamme de services plus étendue, permet de contourner l'obstacle de l'analphabétisme et intègre l'utilisation du wolof, qui joue le rôle de lingua franca au Sénégal.

Fortement influencée par la politique de France Télécom qui, à l'époque, mise sur le Minitel, la Sonatel ne s'intéresse guère au développement d'Internet. Les acteurs de certains segments de la société, opérant notamment dans le monde de l'enseignement supérieur et de la recherche ainsi que dans le milieu des organisations non gouvernementales (ONG), sont obligés de trouver des solutions alternatives pour accéder aux réseaux de messagerie électronique. C'est ainsi que, dès la fin des années 80 , I'ORSTOM ${ }^{11}$ installe un nœud du "Réseau intertropical d'ordinateurs" (RIO) qui donne naissance au premier système de messagerie électronique installé au Sénégal [Renaud, 2000]. Quelques années plus tard, c'est au tour d'Enda Tiers-Monde de mettre en place un nœud du réseau GreenNet, créé par l'Alliance for progressive communication (APC), qui offre des services similaires aux ONG. Ces systèmes fonctionnent sur la base de vacations pour l'envoi et la réception des messages et ne permettent donc pas une communication en temps réel. À l'occasion du Troisième sommet Africain/Africain-Américain en mai 1995 à Dakar, la première connexion permanente à Internet est réalisée, à titre expérimental, avec l'installation d'un lien VSAT d'un débit de $64 \mathrm{Kbps}$. Fortement médiatisée, l'opération rencontre un vif succès et le grand public, qui ne connaissait d'Internet que ce qu'en disaient les médias, prend conscience des opportunités offertes par cet outil.

La Sonatel est alors obligée de revoir sa position et lance, en juillet 1995, un appel d'offres pour la mise en place d'un point d'accès permanent à Internet. En novembre 1995, la pression s'accentue avec l'envoi au Président de la République d'un mémorandum rédigé par un groupe d'universitaires, demandant la connexion du Sénégal à Internet. La question, qui n'était débattue que dans des cercles restreints, gagne la sphère publique et le Président Abdou Diouf annonce, dans son discours de fin d'année à la nation, la connexion du Sénégal à Internet pour le début de l'année 1996. Finalement, en mars 1996, la Sonatel met en service une connexion permanente à Internet via une liaison d'un débit de $64 \mathrm{kbps}$ avec le satellite Intelsat 635 de la société américaine $\mathrm{MCl}$.

\footnotetext{
${ }^{10}$ En dehors des services proposés par les banques, les rares services disponibles étaient les paris sur les courses de chevaux, l'horoscope, les programmes de radio, etc.

${ }^{11}$ Office de la recherche scientifique et technique d'outre-mer devenu Institut de recherche pour le développement (IRD) en 1999.
} 
De la domination politique à la domination économique :

une histoire des télécommunications au Sénégal

Le mois suivant, sa filiale Télécomplus commercialise les premiers abonnements à Internet [Sagna, 2001, p. 13], marquant ainsi les débuts de l'Internet public au Sénégal. Dans un premier temps, les utilisateurs peuvent uniquement se connecter via le réseau téléphonique commuté (RTC) avec des débits ne dépassant pas les $64 \mathrm{kbps}$ mais, en 2003, la Sonatel lance l'ADSL ${ }^{12}$, offrant des débits qui seront progressivement portés de $128 \mathrm{kbps}^{\mathrm{a}} 1 \mathrm{Mbps}^{13}$. Cependant, quinze ans après l'arrivée d'Internet, son taux de pénétration reste faible puisqu'il ne concerne que $15,7 \%$ de la population ${ }^{14}$ confronté qu'il est à des obstacles qui ont pour noms analphabétisme numérique, cherté de l'équipement informatique, faible nombre d'applications et de services utiles aux citoyens, rareté des contenus locaux, etc.

\section{La fulgurante expansion de la téléphonie mobile}

Cependant, l'innovation majeure est sans aucun doute l'introduction de la téléphonie mobile en septembre 1996 avec le lancement du réseau GSM Alizé de la Sonatel. Au départ, le service n'attire guère le grand public car son ticket d'entrée est particulièrement cher. En effet, Alizé propose une formule postpayée qui implique l'achat d'un téléphone portable et le paiement de frais d'abonnement mensuels auxquels viennent s'ajouter les frais de communication. Dans ces conditions, une étude faite par I'Union internationale des télécommunications (UIT) en 1998 prévoyait que le seuil des 30000 abonnés ne serait atteint qu'en l'an 2000 [UIT, 1998b, p. 109]. En réalité, l'année 2000 voit non seulement le nombre de clients franchir la barre des 200000 abonnés mais également dépasser celui de la téléphonie fixe. II faut dire qu'entre temps, en avril 1999, un second opérateur, Sentel, est arrivé sur le marché avec une formule prépayée qui a obligé Alizé à investir ce créneau qu'il avait négligé dans un premier temps. La concurrence ayant fortement fait baisser le prix des abonnements comme celui des communications, la croissance du marché de la téléphonie mobile a pris des proportions impressionnantes avec un million d'abonnés en 2004, trois millions en 2006 et cinq millions en 2008. La concurrence s'est encore accrue à partir de 2009 avec l'arrivée d'Expresso ${ }^{15}$, portant en 2011 le nombre d'abonnés à 9352868 et le taux de pénétration de

\footnotetext{
${ }^{12}$ Le lancement de l'ADSL a été rendu possible par la mise en service, en mai 2002, du câble sousmarin en fibre optique SAT-3/WASC/SAFE reliant le Portugal à la Malaisie, d'une capacité de 120 Gbits par seconde.

${ }^{13}$ La Sonatel propose également une offre à 10 mégabits qui cible plutôt les usages professionnels.

14 UIT, décembre 2011 (http://www.internetworldstats.com/).

${ }^{15}$ Expresso, filiale de l'opérateur soudanais Sudatel, a obtenu une licence globale (fixe, mobile et Internet) en septembre 2007.
} 
Olivier SAGNA

la population à $76,84 \%$ [ARTP, 2011b], pendant que la téléphonie fixe ne compte guère que 346406 abonnés soit un taux de pénétration de 2,85\% [ARTP, 2011a].

La progression du nombre d'abonnés s'accompagne d'une expansion de la couverture géographique des réseaux de téléphonie mobile. C'est ainsi que le réseau, qui ne couvrait à l'origine que Dakar, les principales villes du pays et quelques grands axes routiers, couvre aujourd'hui plus de $90 \%$ de la population et $95 \%$ des villages de plus de 500 habitants [Sonatel, 2011, p. 22]. De plus, nombre d'innovations ont été apportées avec notamment le lancement du GPRS $^{16}$ par la Sonatel en décembre 2005 puis celui de la technologie EDGE ${ }^{17}$ à partir de 2006. En 2010, la $3 G^{18}$ fait son apparition à l'initiative d'Expresso, suivi en 2011 par la Sonatel ${ }^{19}$, autorisant par là même le développement de l'Internet mobile. Parmi les facteurs qui concourent à l'expansion de la téléphonie mobile, il faut également citer les nombreuses promotions organisées par les opérateurs qui contribuent à faire baisser le coût réel des communications ${ }^{20}$. Enfin, le prix des téléphones portables a considérablement chuté et il est désormais possible de trouver des appareils neufs à des prix très abordables sans parler des opportunités offertes par le marché des appareils d'occasion.

Tiré par le sous-secteur de la téléphonie mobile, le secteur des télécommunications occupe désormais une place déterminante dans l'économie sénégalaise, tant du point de vue des investissements consentis, du chiffre d'affaires réalisé, des taxes et impôts versés que des emplois directs et surtout indirects créés et du rôle transversal qu'il joue dans la société. Si l'on se réfère uniquement à la Sonatel, cette société a investi entre 2000 et 2010 près de 961 milliards de FCFA ${ }^{21}$, ce qui en fait le premier investisseur du pays. Son chiffre d'affaires est passé de 126 milliards de FCFA en 2000 à 599 milliards de FCFA en 2010 soit une progression de plus de $375 \%$ en un peu plus d'une décennie. À travers les télécentres privés puis la revente de recharges téléphoniques et la sous-traitance, elle a créé des dizaines de milliers d'emplois. Mieux, elle est devenue un groupe de télécommunications international opérant en Guinée, en Guinée-Bissau et au Mali.

\footnotetext{
${ }^{16}$ La norme GPRS permet la transmission de données par paquets avec des débits réels de l'ordre de 40 à $50 \mathrm{Kbit}$ par seconde autorisant l'envoi et la réception de messages électroniques.

${ }^{17}$ La norme EDGE offre des débits réels de l'ordre de 100 Kbits par seconde qui permettent de naviguer sur Internet.

${ }^{18} \mathrm{La}$ technologie HSDPA autorise des débits réels de l'ordre de 7,2 Mbits par seconde permettant de naviguer sur Internet dans de bonnes conditions.

19 La Sonatel, qui avait expérimenté cette technologie en mars 2008, ne s'est vue accorder une licence 3G par les autorités qu'en mars 2011.

${ }^{20}$ Pour l'achat d'une recharge de crédit téléphonique, des bonus de $50 \%, 100 \%$, voire $200 \%$, sont régulièrement offerts par les opérateurs.

${ }^{21}$ Un euro est égal à 656 FCFA.
} 
De la domination politique à la domination économique :

une histoire des télécommunications au Sénégal

Du point de vue de l'utilisation de ces outils, malgré une fracture numérique réelle recoupant la fracture sociale qui divise la société sénégalaise, le temps est bien loin où ils étaient réservés à une petite minorité. Le téléphone s'est banalisé, Internet est de plus en plus utilisé par l'État, le secteur privé et les citoyens, le Web et les réseaux sociaux sont devenus des extensions de la sphère publique où naissent et se développent toute sorte de débats, y compris les débats politiques, offrant ainsi de nouveaux espaces à la liberté d'expression et aux dynamiques citoyennes. Cela étant, ces éléments « flatteurs » ne doivent pas être l'arbre qui cache la forêt, à savoir la capture du secteur des télécommunications par les multinationales étrangères à l'occasion de sa libéralisation.

\section{Quand libéralisation rime avec dépossession}

Bien gérée, non déficitaire, ayant contribué à démocratiser l'accès au téléphone notamment grâce aux télécentres [Sagna, 2009], même si la question de la téléphonie rurale restait irrésolue [Thiam, 2004], la Sonatel n'a pourtant pas échappé à la vague de privatisations lancée au milieu des années 80 . Suite à la signature de l'Accord général sur la commercialisation des services (AGCS) en 1994, les autorités sénégalaises, dont la politique économique était largement dictée par la Banque mondiale (BM) et le Fonds monétaire international (FMI) dans le cadre d'un Plan d'ajustement structurel (PAS), ont adopté, en 1995; une loi permettant la privatisation de la Sonatel ${ }^{22}$. Elle a été complétée par l'adoption d'un nouveau Code des télécommunications ${ }^{23}$ qui a introduit une concurrence limitée dans le secteur de la téléphonie mobile et une concurrence totale dans celui des services à valeur ajoutée. En 1996, un appel d'offres international pour la sélection d'un " partenaire stratégique » a abouti à la cession de $33 \%$ du capital de la Sonatel à France Télécom pour la somme de 70 milliards de FCFA, 17,6 \% étant vendus au grand public et à des investisseurs institutionnels, $10 \%$ réservés aux travailleurs et retraités de la société et $5 \%$ à un opérateur africain [Sagna, 2010]. Cependant, en 1999, l'État vend $9 \%$ de ses actions à France Télécom et voit sa part dans le capital de la Sonatel passer à $27,67 \%$, perdant ainsi toute capacité d'exercer sa minorité de blocage au sein du conseil d'administration (Plane, 2002, p. 10) et de peser sur les choix stratégiques.

${ }^{22}$ Loi no 95-25 du 29 août 1995 modifiant l'annexe de la loi n 87-23 du 18 août 1987.

${ }^{23}$ Loi n $96-03$ du 26 février 1996 portant création du Code des télécommunications. 
Dans le sillage de cette privatisation, la prise de contrôle progressive du secteur des télécommunications par des firmes étrangères n'a fait que s'accentuer au fur et à mesure que s'approfondissait le processus de libéralisation de l'économie. Ainsi, en 1998, le gouvernement a accordé une licence de téléphonie mobile à Sentel, société dont le capital était détenu à $75 \%$ par la firme luxembourgeoise Millicom International Cellular (MIC) et à $25 \%$ par un investisseur privé sénégalais. Cependant, en mars 2006, MIC a racheté les parts de son partenaire, devenant ainsi une société à capitaux et intérêts entièrement étrangers. L'octroi de la troisième licence de télécommunications a renforcé la tendance à l'exclusion des nationaux du secteur des télécommunications, pourtant l'un des plus rentables de l'économie et l'un des plus stratégiques pour le pays. En effet, malgré la mobilisation de l'Organisation des professionnels des TIC (OPTIC) qui avait exigé que l'État lui réserve au moins $51 \%$ des parts du capital de l'opérateur devant être sélectionné, elle n'en obtiendra finalement que $15 \%$ bien qu'ayant entre-temps ramené ses prétentions à $30 \%$ [Fall, 2007].

La reprise en main du secteur des télécommunications par des firmes étrangères ne s'est pas arrêtée là. Elle s'est également attaquée aux symboles que représentent les marques commerciales utilisées localement par les opérateurs. Ainsi, la marque commerciale Hello, sous laquelle Sentel avait développé ses activités au Sénégal depuis avril 1999, a-t-elle été abandonnée, en novembre 2005, au profit du label Tigo utilisée par MIC à travers le monde. Confirmant cette tendance à l'uniformisation des marques au nom de la globalisation, le nom de l'opérateur Sentel, officiellement titulaire de la licence de téléphonie mobile, a progressivement disparu dans la politique de communication de l'entreprise au profit de Tigo. Sur un plan subjectif, cela a entraîné la dilution du caractère "national " de l'opérateur puisque le vocable Sentel associait, aussi bien en français qu'en wolof, les notions "Sénégal » et " télécommunications". Une année plus tard, ce même processus a été mis en œuvre par France Télécom avec le remplacement des marques commerciales Alizé (téléphonie mobile), Sentoo (Internet) et Keurgui TV (télévision sur Internet) par la marque Orange [Top, 2006]. Le plus cocasse dans cette affaire est que la Sonatel doit désormais s'acquitter de "branding fees " pour être autorisée à utiliser la marque Orange qui lui est imposée par sa maison mère !

Toute marque à connotation nationale ayant disparu du paysage des télécommunications, la firme soudanaise Sudatel n'a même pas essayé d'utiliser une marque faisant "couleur locale" lors de son entrée sur le marché sénégalais en janvier 2009, mais s'est contentée d'utiliser Expresso, label utilisé par toutes les filiales étrangères du groupe. 
De la domination politique à la domination économique :

une histoire des télécommunications au Sénégal

Cependant, la perte d'identité que constitue la substitution de labels " globaux " aux marques locales ne constitue qu'un épiphénomène au regard du processus beaucoup plus fondamental de dépossession et de domination économique qui s'est opéré au nom de la privatisation des opérateurs historiques et de la libéralisation de l'économie. La principale conséquence de ces réformes est d'avoir fait entièrement passer le secteur des télécommunications sous la coupe d'opérateurs étrangers et principalement sous celle de l'opérateur historique de l'ancienne puissance coloniale [Sagna, 2010]. D'ailleurs, à l'occasion de la tentative de l'État sénégalais de céder 9,87 $\%$ de ses actions à France Télécom qui aurait alors détenu $52,2 \%$ du capital de la Sonatel, les syndicats de travailleurs n'ont pas hésité à dénoncer une tentative de « recolonisation » des télécommunications [AFP, 2009].

\section{Conclusion}

Cent cinquante ans après la construction de la première ligne télégraphique pour les besoins de la domination coloniale, les télécommunications jouent un rôle majeur dans le processus de développement politique, économique, social et culturel du Sénégal. Jadis réservées à une minorité, elles sont désormais utilisées par la plupart des secteurs d'activité et catégories sociales. L'infrastructure, encore embryonnaire à la fin de l'ère coloniale, a été considérablement étendue et modernisée à travers la mise en œuvre de politiques publiques qui ont accordé une haute priorité au secteur. Les télécommunications spatiales ont été introduites, les connexions au réseau mondial des câbles sous-marins multipliées, le réseau national numérisé et une large gamme de services proposée. Les télécentres puis la téléphonie mobile ont démocratisé l'accès au téléphone, les cybercafés puis l'Internet mobile ont popularisé l'utilisation d'Internet. Contribuant pour plus de $7 \%$ au PIB, les télécommunications sont devenues l'un des moteurs de la croissance du pays et I'Internet et les réseaux sociaux jouent un rôle croissant dans la sphère publique et dans la vie quotidienne des Sénégalais et des Sénégalaises. Cependant, du fait des politiques libérales dictées par les bailleurs de fonds, ce secteur, tout aussi stratégique que rentable, échappe désormais au contrôle des nationaux et les richesses qu'il génère profitent essentiellement aux multinationales. Jadis outil de la domination coloniale, les télécommunications se sont donc transformées en l'un des principaux instruments de la domination multiforme imposée au Sénégal dans le cadre de la mondialisation capitaliste. 
Olivier SAGNA

\section{Références bibliographiques}

AGENCE FRANCE PRESSE (AFP), 2009, « Mobilisation syndicale contre une « recolonisation » des télécoms », AFP, 29 avril 2009.

AGENCE DE REGULATION DES TELECOMMUNICATIONS ET POSTES (ARTP), 2011a, Observatoire de la téléphonie fixe, décembre 2011, Dakar.

AGENCE DE REGULATION DES TELECOMMUNICATIONS ET POSTES (ARTP), 2011b, Observatoire de la téléphonie mobile, décembre 2011, Dakar.

ASSOCIATION DES AMIS DES CABLES SOUS-MARINS (AACSM), 2006, Une chronologie des évènements des télécommunications (1800-2005). $<$ http://www.cablesm.fr/joomlacablesm/images/stories/bulletins/chronologie.p $\underline{d f>}$, dernière consultation le 16 février 2011.

DAFFE G. et DANSOKHO M., 2002, « Les nouvelles technologies de l'information et de la communication : défis et opportunités pour l'économie sénégalaise, » in : DIOP M.C. (dir.), Le Sénégal à l'heure de l'information, Paris : Karthala, pp 45-96.

DESBOIS D., 2000, « Les politiques du développement dans le secteur des télécommunications », in : CHENEAU-LOQUAY A. (dir), Enjeux des technologies de l'information et de la communication en Afrique, Paris : Karthala, pp. 129-149.

ERNE R. 2007, Les télécommunications spatiales et les ressources de l'espace extra-atmosphérique : l'évolution de leur réglementation. Université de Genève. Institut universitaires des études internationales. Thèse en sciences politique.

FALL A., 2007, « $15 \%$ du capital de Sudatel aux nationaux : Les privés s'en prennent à l'Artp », Le Quotidien (Dakar), 19 septembre 2007.

FALL C., 1964, "Les télécommunications outre-mer : Sénégal », Industries et travaux d'outre-mer, $\mathrm{n}^{\circ} 127$, juin 1964, pp 487-489.

FIELD A. J., 1994, "French Optical Telegraphy, 1793-1855": Hardware, Software, Administration in Technology and Culture, Vol. 35, $\mathrm{n}^{\circ}$ 2, pp 315347.

FIGUIER L., 1849, La Revue des deux mondes, $\mathrm{n}^{\circ} 3$, pp 594-622.

GOUVERNEMENT GENERAL DE L'AFRIQUE OCCIDENTALE FRANCAISE, 1907, Les postes et télégraphes en Afrique occidentale, Corbeil : Éditions Crété. 
De la domination politique à la domination économique : une histoire des télécommunications au Sénégal

HACHMANIAN M., 2006, FCR et l'Afrique (période 1960/1990). <http://anciensdefcr.eu/histoire/fcr afrique/fcr et afrique.htm>, dernière consultation le 16 février 2011.

KANE O., 2010, L'organisation des télécommunications au Sénégal. Entre gouvernance et régulation, Paris : Karthala.

LEMESLE R-M., 2002, L'économie des télécommunications en Afrique, Paris : Agence universitaire de la Francophonie/Karthala.

LOMBARD J., 2006, «Croître ou dépérir. Lieux intégrés, lieux oubliés sur l'axe Dakar-Mali » in : CHALEARD J-L., CHANSON-JABEUR C. et BERANGER C. (dir.), Le chemin de fer en Afrique, Paris : Karthala, pp 68-86.

LY A., 1958, La Compagnie du Sénégal, Paris : Présence africaine.

MBAYE S., 1980, « Commerce et politique à Saint-Louis de 1758 à 1858 », Ethiopiques, $\mathrm{n}^{\circ} 24$, octobre 1980, pp 86-97.

NIANG B., 1977, Essai sur l'histoire du courrier postal et des lignes télégraphiques au Sénégal (1850-1900), mémoire de maîtrise d'histoire, Université de Dakar.

PLANE P., 2002, Privatisation et ouverture des télécommunications en Afrique subsaharienne : modalité et implications des réformes, Clermont Ferrand : CERDI.

RENAUD P., 2000, « Historique de l'Internet du Nord au Sud » in : Enjeux des technologies de la communication en Afrique, CHENEAU-LOQUAY A. (dir.) Paris : Karthala, pp 91-99.

ROYER P., 1969, Géographie des postes et télécommunications au Sénégal, mémoire de maîtrise de géographie, Université de Dakar.

SAGNA O., 2001, Les technologies de l'information et de la communication et le développement social au Sénégal : un état des lieux, Genève : UNRISD.

SAGNA O., 2008, "Le Sénégal dans l'ère de l'information (1996-2006 », NETCOM, vol. 22, n 1 \& 2 et Netsuds, vol. 3, pp 13-36.

SAGNA O., 2009, "Les télécentres privés du Sénégal : la fin d'une « success story »", Netsuds, $\mathrm{n}^{\circ} 4$, pp 27-43.

SAGNA O., 2010, " Privatisation, libéralisation, régulation : La réforme des télécommunications au Sénégal », Afrique contemporaine, $\mathrm{n}^{\circ} 234$, pp 113126. 
SONATEL, 2010, Résultats 2010, Dakar : Sonatel.

SY J. H., 1996, Telecommunications dependency: The African Saga (18501980), Dakar ; Alternative Communication Inc.

THIAM B., 2004, Étude sur la téléphonie rurale au Sénégal, Dakar : Institut Panos Afrique de l'Ouest.

THIAW A. E., 1995, Infrastructures et services de télécommunications au Sénégal : le développement de la téléphonie, mémoire de maîtrise de géographie, Université Cheikh Anta Diop de Dakar.

TOP A., 2006, « La mondialisation en marche : Orange s'impose au Sénégal », Batik (Dakar), n 88, novembre 2006.

UNION INTERNATIONALE DES TELECOMMUNICATIONS (UIT), 1998a, Étude de cas sur l'évolution de l'environnement international des télécommunications : Sénégal, Paris : ICEA.

UNION INTERNATIONALE DES TELECOMMUNICATIONS (UIT), 1998b, Indicateurs des télécommunications africaines 1998, Genève : UIT.

WALLESTEIN I., 1985, Le capitalisme historique, Paris :Ed. La Découverte.

WESLEY JOHNSON G. Jr., 1991, Naissance du Sénégal contemporain. Aux origines de la vie politique moderne (1900-1920), Paris : Karthala.

ZIMMERMANN M., 1900, "Projet d'un réseau français de câbles sousmarins », Annales de géographie, t. 9, n 48 pp. 469-470. 